\title{
Experimental Spinal Cord Trauma A Biomechanical Viewpoint
}

\author{
Manohar M. Panjabi, Ph.D., Dr.Tech. \\ Yale University School of Medicine, New Haven, Connecticut, 06510, U.S.A.
}

Animal models of spinal cord injury have been developed to study the clinical problem associated with spinal cord trauma. First and foremost they provide basic understanding of the phenomena of mechanical trauma of the cord and the resulting neurological dysfunction. Secondly, they provide the means by which old and new treatment modalities may be tried out in a laboratory setting under controlled conditions before they are used clinically. For a model to be useful, it must mimic the central aspects of the clinical situation, be reproducable with a high degree of certainty, and be able to be adapted, without too much cost and time-loss, to simulate different aspects of the clinical problem. Since the time it was introduced, the method of Allen (1911), or some modification of it, has become the model most often used for the study of spinal cord trauma.

The method of Allen is also referred to as the Weight Drop Method. In its simplest form it consists of dropping a weight of known mass from a known height onto an exposed spinal cord. Generally, an impounder, a cylindrical piece made of metal or plastic aligned vertically, is interposed between the falling weight and the spinal cord so as to precisely localise the area of impact. The weight is guided by either a rod or a tube during its free fall. The animal is supported either directly on its chest on the table or suspended from the spinous processes adjacent to the laminectomy. To produce different severities of trauma, either different weights are dropped from the same height or a single weight is dropped from different heights, or, less commonly, a combination of the two procedures is used. Originally, it was suggested that the severity of the trauma may be quantified by the 'product of the weight in grams and the height in centimeters' (Allen, 1911). This was expressed in 'gm-cm'. The simplicity of this measure of spinal cord trauma is equalled by its popularity over the last 75 years. However, this simplicity is false and misrepresents the complex biomechanical interaction that takes place between the falling weight, the impounder, and the spinal cord and the underlying supporting structure during the short duration of trauma. We will look into some of these interactions and the difficulties and variability inherent to this method of quantifying trauma and also the various modifications that have been proposed and tried out in some recent studies. 
The main problem with the model of Allen has been the variability in the trauma observed by (a) different researchers using the same apparatus and a given gm-cm of trauma, and (b) different research groups using apparently similar apparatus and procedure. In the first case two researchers within the same research group may use the same apparatus and a constant $\mathrm{gm}-\mathrm{cm}$ of trauma but may use different combinations of weight and height to obtain the given gm-cm of impact. The constancy of gm-cm implies that the falling weight has a given potential energy at the start of the fall and equal amount of kinetic energy (due to its velocity) at the time of impact with the spinal cord (assuming that the air resistance is negligible). It may still produce different traumas depending on specific combination of weight and height. This idea was first suggested in 1976, Dohrmann \& Panjabi (1976) and validated experimentally. Since then it has been confirmed by other researchers (Molt et al., 1979).

The second problem mentioned above of different research groups, using precisely the same weight and height combination but different apparatus, is more complex. The variability in this case comes basically from two factors: the variation in mass, material and contact area of the impounder; and the variability in the underlying supporting structure, i.e. the suspension of the spinal column of the animal. The 'mass' aspect of the first factor has been explored theoretically. The impounder mass affects the energy transfer from the weight to the cord (Dohrmann \& Panjabi, 1976). One may assume that the contact area between the impounder and the spinal cord would be an important factor in the trauma delivered to the cord. However, this does not seem to be a critical factor. In an experiment three groups of animals were traumatised, each with a different contact area of the impounder, all other factors being the same. The results showed that the variation in the contact area did not produce significant variation in lesion size of the spinal cord (Molt et al., 1979). The second factor is extremely important but has not been explored. We know from our daily experience that the magnitude of the force applied to an object is directly related to the resistance the object offers to the force. This is Newton's third law: reaction is equal and opposite to the action. Therefore, in general a flexible suspension will result in a lower force delivered to the cord as compared to that due to a stiff suspension.

To alleviate these difficulties several approaches have been pursued. The main reason that the $\mathrm{gm}-\mathrm{cm}$, as a measure of impact delivered to the spinal cord, is imprecise is due to the fact that the energy contained in the falling weight at the time of impact is not completely transformed into the traumatic energy transferred to the spinal cord. The energy transfer at the time of impact is dependent upon the masses, physical properties and velocities of the contacting bodies: falling weight, impounder, spinal cord and spinal column. This is a complex mechanical phenomenon which is not easily understood and quantified. For this reason other biomechanical parameters have been suggested as replacement for the $\mathrm{gm}-\mathrm{cm}$. The force applied to the spinal cord would be a logical parameter to consider to define the trauma actually delivered to the cord. Strain gauged impounders, i.e. impounder with built in load transducers, have been designed and used to do precisely this. Good correlations of the measured force in newtons with the spinal cord lesion and, more importantly, with the motor dysfunction have been obtained (Daniell et al., 1975; Dohrmann \& Panjabi, 1976; Molt et al., 1979; Panjabi \& Wrathal, 1987). Although force is an excellent 
mechanical parameter, it tells only part of the story. We know from experience that the duration of the application of the force is as important as the magnitude of the force. For example, when you want to push a car on the road, often a small force (applied by hands) acting for a longer period of time produces better results than a large force (a blow with a hammer) acting for a shorter period of time. Thus, the traumatic energy imparted to and the lesion produced in the spinal cord are functions of the force as well as the time duration of its application. A mechanical entity that takes into account both the factors is called impulse. This is defined as the force applied multiplied by its time duration of application. Therefore, if we measure the force-time curve during the impact, then it is possible to compute the impulse given to the spinal cord. In practical terms this is the area under the force-time curve and has units of newtonseconds. It has been shown experimentally that this is a better measure of the trauma than the force alone. The correlation co-efficients for the impulse with the cord lesion and the neurological dysfunction as represented by motor deficit score are very respectable indeed reaching values of 0.8 (Panjabi \& Wrathal, 1987).

There is another mechanical parameter of equal importance representing the other half of the story, namely the deformation of the cord. It has been argued by some that the deformation of the cord is probably more important than the measurement of the force or even impulse (Hung et al., 1975; Anderson, 1982). Of course, the two parameters of force and cord deformation are not really independent but are related to each other via the physical properties of cord, dura etc.

There are basically two methods of measuring the deformation of the cord. The high speed movie camera may be used to monitor the cord visually from the lateral direction during the impact. The deformation produced as a function of time can then be obtained by studying the sequential frames and measuring the cord diameter (Hung et al., 1975; Kooze Kanan et al., 1976). Of course, this method requires a very high speed movie camera (total trauma duration is only 7 thousandths of a second) and provides only discrete information. In contrast the second method produces continuous data and uses either a deformation transducer to monitor or a power-activated device to produce the deformation (Dohrmann \& Panjabi, 1976; Dohrmann et al., 1978; Anderson, 1982). The deformation-time signal so obtained, may be differentiated to provide, in addition, the velocity-time signal. Finally, one may record both the force and deformation signals using transducers to obtain the energy-time signal (Panjabi et al., 1977). As the trauma produced in the spinal cord is due to the dissipation of the energy, it seems logical to consider the energy transmitted into the cord as a measure of injury.

The output parameters of the spinal cord trauma model have varied. Spinal cord lesion has been quantified by its length, cross-sectional area and/or the total volume. The best correlations with the biomechanical parameters, such as force and impulse, have been found with the lesion volume (Dohrmann \& Panjabi, 1976; Noble \& Wrathal, 1985). The second output parameter has been the sensory evoked potential (SEP) (Bohlman et al., 1981; Anderson, 1982; Raines et al., 1986). Finally, the most important output parameter is that of motor function or loss of it, first proposed by Tarlov (1954). This has been 
subsequently modified and additional parameters reflecting overall behaviour of the animal have been added (Gale et al., 1985). Respectable correlation coefficients of up to $0 \cdot 8$ have been obtained between the biomechanical parameters of trauma, such as the impulse, and the functional parameters, such as the motor function and behavioural scores (Panjabi \& Wrathal, 1987).

Better understanding of spinal cord injury has been obtained using animal models in which a mechanical trauma is produced, most often by a simple weight drop method, and the output is measured by lesion volume, SEP and/or motor function loss. By standardising the equipment and providing a better definition of the trauma highly significant quantitative relationships have been obtained between the biomechanical parameters and the functional losses in an animal model. Further refinements and standardisations of the apparatus and validation studies are needed to study other biomechanical parameters such as deformation and energy. One of the significant uses of this spinal cord trauma model remains that animals with a predefined amount of neurological deficit may be produced with high reproducibility and certainty so that different treatment modalities, old as well as new, may re evaluated.

\section{Acknowledgements}

Work supported in part by National Institute of Health Research Grants AM 30361, AM 34699 and a Veterans Administration Research Grant.

\section{References}

ALLEN AR 1911 Surgery of experimental lesion of spinal cord equivalent to crush injury by fracture dislocation of spinal column: A preliminary report. Fournal of the American Medical Association 57:878-880

ANDERSON TE 1982 A controlled pneumatic technique for experimental spinal cord contusion. Fournal of Neuroscience Methods 6:327-333

Bohlman HH, Bahniuk E, Field G, Raskulinecz G 1981 Spinal cord monitoring of experimental incomplete cervical spinal cord injury. Spine 6: 428-436

DANIELl HB, FRANCIS WW, LEE WA, et al. 1975 Method of quantitating injury inflicted in acute spinal cord studies. Paraplegia 13:137-197

Dohrmann GJ, Panjabi MM: 1976 Standardized spinal cord trauma: Biochemical parameters and lesion volume. Surgical Neurology 6:263-477

Dohrmann GJ, Panjabi MM, Banks D 1978 Biomechanics of experimental spinal cord trauma. fournal of Neurosurgery 48:993-1001

Gale K, KeRAsidis H, WRATHALl J 1985 Spinal cord contusion in the rat behavioral analysis of functional neurological impairment. Experimental Neurology 88:123-134

Hung TK, ALBIN MS, BRown TD, et al. 1975 Biomechanical responses to open experimental spinal cord injury. Surgical Neurology 4:271-276

KoOZEKANANi SH, Vise WM, HASHEMI RM, et al. 1976 Possible mechanisms for observed pathophysiological variability in experimental spinal cord injury by the method of Allen. Fournal of Neurosurgery 44:429-434

Molt JT, Nelson LR, Poulos DA, et al. 1979 Analysis and measurement of some sources of variability in experimental spinal cord trauma. Fournal of Neurosurgery 50:784-791

Noble L, Wrathall J 1985 Spinal cord contusion in the rat: Morphoenetric analyses of alternations in the spinal cord. Experimental Neurology 88:135-149

PANJABI MM, Dicker DB, Dohrmann GJ 1977 Biomechanical quantification of experimental spinal cord trauma. Fournal of Biomechanics 10:681-687

PANJABi MM, WRAThall JR. Biomechanical analysis of spinal cord injury and functional loss. In press

Raines A, Dretchen KL, Marx K, Wrathall J 1986 Somatosensory evoked potentials as an index of spinal cord injury in the rat. Society of Neuroscience Abstract, in press

TARLOV IM 1954 Spinal cord compression studies. III. Time limits for recovery after gradual compression in dogs. Archives of Neurology and Psychiatry 71:588-597 